\title{
ROBUST ROAD SIGNS SEGMENTATION IN COLOR IMAGES
}

\author{
Bishesh Khanal, Sharib Ali and Désiré Sidibé \\ Université de Bourgogne, Laboratoire Le2i, UMR CNRS 5158, 12 rue de la Fonderie, 71200, Le Creusot, France \\ bisheshkh@gmail.com,ali.sharib2002@gmail.com,dro-desire.sidibe@u-bourgogne.fr
}

\begin{abstract}
Keywords: Road sign detection; Color segmentation; Color constancy; Log-chromaticity color space.
Abstract: $\quad$ This paper presents an efficient method for road signs segmentation in color images. Color segmentation of road signs is a difficult task due to variations in the image acquisition conditions. Therefore, a color constancy algorithm is usually applied prior to segmentation, which increases the computation time. The proposed method is based on a log-chromaticity color space which shows good invariance properties to changing illumination. Thus, the method is simple and fast since it does not require color constancy algorithms. Experiments with a large dataset and comparison with other approaches, show the robustness and accuracy of the method in detecting road signs in various conditions.
\end{abstract}

\section{INTRODUCTION}

The detection and identification of road signs is an important component of any Intelligent Transportation System (ITS). It is useful in highway maintenance, sign inventory or driver support systems. A full road sign recognition system is composed of two main stages, detection and recognition (Bascon et al., 2007). The goal of the first stage is to identify potential road signs in the image, i.e. the possible regions that represent road signs characteristics, using either color or shape information. In the second stage, the potential regions are further analyzed to determine the correct sign and its meaning.

In this paper we focus on the detection stage of road signs in still images or videos. Road sign detection in natural scene images is a challenging task due to the complexity of the scene's environment. The challenges include changes of illumination, shadows, occlusions, size variation, and the presence of object similar in color.

Color and shape are the two main features used to detect and recognize road signs. Color as a low-level feature offers many advantages such as robustness to occlusions, scale variation and geometric transformations. However, the color of an object depends on the illumination conditions, the camera parameters and the reflectance properties of the object. A good road signs detector must then be robust against illumination variations in uncontrolled environment and a color constancy algorithm is usually used as a preprocessing step. However, this increases the compu- tation time of the detection method.

In this paper, a fast and robust road sign detection method based on color segmentation is proposed. The method is based on the invariance properties of the log-chromaticity color space. The log-chromaticity color space shows two important properties. Firstly, a surface color seen under different illuminant colors tends to lie on a straight line in this space. Secondly, for a given camera, all these lines are parallel to each other for different surface colors. Thus an illumination invariant representation of images can be obtained in log-chromaticity color space (Finlayson et al., 2004). We take advantage of this invariant property to develop an efficient road signs segmentation algorithm which is robust against illumination variations and shadows. This robust and fast algorithm can be used as main input for a shape recognition and road signs interpretation module of an ITS.

The paper is organized as follows: Section 2 describes previous work on road signs segmentation based on color information. Section 3 introduces the proposed method for road signs detection, and experimental results are shown in Section 4. Finally, some conclusions are derived in the last section.

\section{RELATED WORK}

The two main features used to detect road signs are the color (red, yellow, blue, green, and white) and the shape (circle, triangle, rectangle, and octagon) of road signs. Road signs are specifically designed to 
have a distinctive color from the surrounding environment in order to help drivers to recognize them easily and quickly. Therefore, several detection methods are based on color segmentation. Among different segmentation approaches, image thresholding is the simplest method and is used in a lot of works. For example, (Benallal and Meunier, 2003) use a simple threshold formula applied to the red color channel to detect red road signs in RGB color space. However, RGB space is very sensitive to illumination changes and traffic scenes tend to have varying illumination. Therefore, a color space transformation from RGB space to another color space is usually performed. Several color spaces are used including HSV (Paclik et al., 2000), HSI (Escalera et al., 2003; Fang et al., 2003), CIECAM97 (Gao et al., 2006) and IHSI (Fleyeh, 2004). The main assumption in these works is that the hue and saturation components are less sensitive to changes in illumination compared to the luminance component. Hence, the luminance is discarded in the segmentation algorithm. Nevertheless, most of the color based methods can deal only with slight variations in lighting conditions.

(Fleyeh, 2004) compares three segmentation methods for road signs detection in the Improved Hue, Luminance and Saturation (IHLS) color space. The first method uses an adapted threshold which is computed for each image based on its mean luminance value. The second method uses region growing, and the third method, similar to the method developed by (Escalera et al., 2003), is based on lookup tables where the thresholds are obtained from a set of training images. The author concludes that the segmentation method using adaptive threshold gives the best results. Though this method, using dynamic thresholding can adapt to the image content, it fails to correctly segment road signs in different lighting conditions. For example, it cannot detect a road sign acquired by night, in a raining day or in very bright sunlight.

To achieve robustness against illumination changes, a color constancy algorithm can be used as a preprocessing step. For example, (Fleyeh, 2005) uses the local color shifts method of (Ebner, 2004) to normalize the images before applying the dynamic thresholding method. (Le et al., 2010) use the same color constancy algorithm and (Zakir et al., 2011) use the Gray-World algorithm to normalize the images. In the experiments reported by the authors of these work, applying a color constancy algorithm leads to an improvement in the detection accuracy. Nevertheless, using color constancy also increases the overall computation time of the detection method and, thus, limits its application for real-time systems.
In this paper, we present a robust road signs segmentation method which achieves very good detection results while avoiding the use of color constancy. The method is based on the invariance properties of the log-chromaticity color space.

\section{ROBUST ROAD SIGNS SEGMENTATION}

\subsection{Log-Chromaticity Color Space Properties}

The log-chromaticity color space (LCCS) is a 2D space obtained by taking the logarithm of ratios of color channels. For example, $\log (\mathrm{R} / \mathrm{G})$ and $\log (\mathrm{B} / \mathrm{G})$ form a $2 \mathrm{D}$ LCCS. The illumination invariance property is based on the image formation model given by:

$$
I_{c}=\sigma \int_{\Omega} E(\lambda) S(\lambda) Q_{c}(\lambda) d \lambda, c \in\{R, G, B\} ;
$$

where, $I_{c}$ is the color intensity at a pixel for the color channel $c, \sigma$ is the Lambertian shading, $E$ is the illumination power spectral distribution, $S$ is the surface spectral reflectance function and, $Q_{c}$ is the camera sensor sensitivity function. The integral is computed over the visible spectrum $\Omega$.

Under the assumptions of Planckian lighting, Lambertian surface and a narrowband camera, equation 1 becomes:

$$
I_{c}=\sigma I k_{1} \lambda_{c}^{-5} e^{-\frac{k_{2}}{T \lambda_{c}} S\left(\lambda_{c}\right) q_{c}}
$$

where $T$ is the illuminant temperature and $k_{1}$ and $k_{2}$ are constants containing the Planck constant, the Boltzman constant and the speed of light in vacuum (Finlayson et al., 2004).

If, we consider the chromatic components by taking the ratios, for example, of Red and Blue channels w.r.t. Green channel, then, from equation 2 it is clear that intensity and shading information are removed. To remove the nonlinearity, we take the natural logarithm of the ratios and obtain:

$\rho_{c}=\log \left(I_{c} / I_{G}\right)=\log \left(s_{c} / s_{G}\right)+\left(e_{c}-e_{G}\right) / T, c \in\{R, B\} ;$

with $s_{c}=k_{1} \lambda_{c}^{-5} S\left(\lambda_{c}\right) q_{c}$ and $e_{c}=-k_{2} / \lambda_{c}$.

This last equation shows that in the LCCS all the color values of a surface seen under different illuminants fall on a straight line. The direction of this line, given by the vector $\left(e_{c}-e_{G}\right)$, is independent of the surface reflectance function. As a consequence, different surface characteristics will produce different lines in the LCCS. However, all these lines are parallel, since they share the same slope (independent from 
the surface). An invariant image can be formed by projecting the chromatic components into a direction orthogonal to these parallel lines.

\subsection{Road Signs Detection in Log-Chromaticity Color Space}

As stated in Section 2, an important characteristic of any road signs segmentation method is its ability to achieve correct detection in the presence of varying illumination conditions.

The invariance properties of the LCCS can be exploited to develop a robust road signs detector. The motivation of our approach is that a characteristic color of road signs, red for instance, under different lighting conditions tend to form a distinct cluster in the LCCS. Indeed, intensity and shading information are removed when projecting the color of a pixel in LCCS. Furthermore, when the illumination is varied, the color values of a surface moves on a straight line in LCCS. On this line, the red color for instance, will occupy a small range. Hence, we can define a simple segmentation rule based on the boundaries of the cluster formed by red signs color in LCCS. The boundaries are obtained from a training set of pixels that are manually selected from various road signs images chosen in order to represent different lighting conditions.

A pixel in an image is classified as a road sign pixel if its projection in the LCCS satisfies:

$$
a \leq \log (R / G) \leq b \text { and } c \leq \log (B / G) \leq d,
$$

where $(a, b, c, d)$ are the thresholds. For red signs, for instance, these thresholds are found to be equal to $(a, b, c, d) \equiv(0.5,2.1,-0.9,0.8)$.

The experiments described in Section 4 show that this simple rule leads to very good detection results in various conditions, providing an efficient solution for road signs segmentation.

\section{EXPERIMENTS}

To evaluate the performance of the proposed road signs segmentation method we apply it on a dataset of 389 images containing 567 road signs of various shapes and types. The dataset is a subset of the one used by (Bascon et al., 2007) and shows images of road signs in different conditions such as bright sunlight and electric lighting by night. We also compare our method with the dynamic thresholding method (Fleyeh, 2005) and its combination with two popular color constancy algorithms: the Gray-World method (Barnard et al., 2002) and the comprehensive
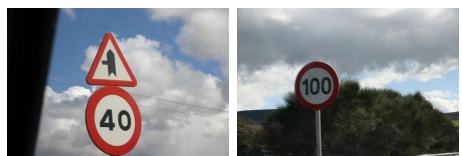

(a)
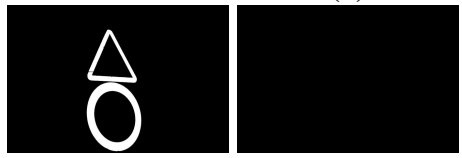

(b)
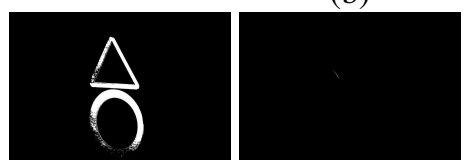

(c)
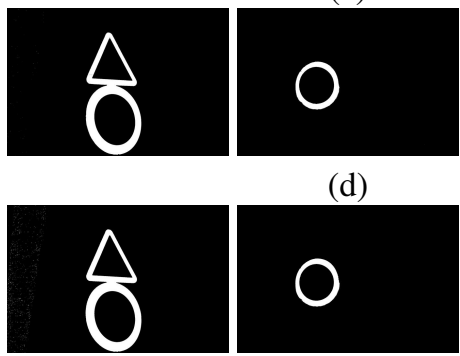

(d)

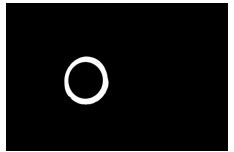

(e)
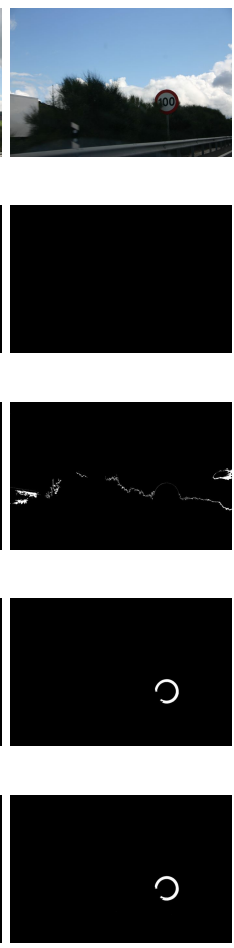

Figure 1: Example of segmentattion results. (a) Original images. (b) Segmentation with the dynamic thresholding method. (c) Segmentation with dynamic threshold and Gray-World constancy. (d) Segmentation with dynamic threshold and comprehensive normalization color constancy. (e) Segmentation with the proposed method.

normalization color constancy algorithm (Finlayson et al., 1998).

Figure 1 shows some segmentation results obtained with the different methods. As can be seen in figure 1(b) and figure 1(c), the dynamic thresholding method and its combination with the GrayWorld color constancy algorithm perform poorly. Some clearly visible road signs are not detected. On the contrary, the use of the comprehensive normalization color constancy algorithm together with dynamic thresholding, figure $1(\mathrm{~d})$, and the proposed method based on log-chromaticity color space, figure 1(e), give very good results. However, the proposed method does not need any color constancy algorithm and is, thus, much faster.

Detection results using the entire dataset are summarised in Table 1. From the 567 road signs, 552 are correctly detected by the proposed method which results in a correct detection rate of more than $97 \%$. The standard dynamic thresholding method achieves very poor performance as it correctly detects less than half of the road signs (correct detection rate of $46 \%$ ). Adding the Gray-World color constancy algorithm 
Table 1: Summary of detection results.

\begin{tabular}{|c|c|c|}
\cline { 2 - 3 } \multicolumn{1}{c|}{} & $\begin{array}{c}\text { Detection rate } \\
(\%)\end{array}$ & $\begin{array}{c}\text { Computation } \\
\text { time per image (s) }\end{array}$ \\
\hline DT & 46.2 & 0.249 \\
\hline DT + GW & 52.02 & 0.546 \\
\hline DT + CN & 87.83 & 29.01 \\
\hline LCCS & 97.35 & 0.093 \\
\hline
\end{tabular}

DT = dynamic thresholding

$\mathrm{DT}+\mathrm{GW}=$ dynamic threshold with Gray-World color constancy

$\mathrm{DT}+\mathrm{CN}=$ dynamic threshold with comprehensive normalization color constancy

LCCS $=$ proposed method in log-chromaticity color space.

slightly improves the results, from $46 \%$ to $52 \%$, while increasing the computation time by a factor two. The comprehensive normalization color constancy algorithm gives good results with a correct detection rate of more than $87 \%$. However, the processing time of comprehensive normalization is very high, which limits its use in real-time applications. On the contrary, the proposed method based on log-chromaticity color space achieves the best detection results in the least computation time as shown in Table 1 . Note that the given computation time are obtain with non optimized MATLAB codes using a $2.4 \mathrm{GHz} \mathrm{CPU}$ and images of size 640 x 480.

The proposed method fails to correctly detect road signs when the image is acquired in complete dark condition, the road sign being only illuminated by the headlights of the moving car. In such a case, all methods fail to detect the road signs.

\section{CONCLUSION}

In this paper, an efficient and robust road signs segmentation method based on color information is proposed. The method is based on finding boundaries of clusters formed by distinctive road signs color in the log-chromaticity color space. Based on invariance properties of this color space, we propose an efficient segmentation method and experiments with a large dataset show that the proposed method is particularly robust against severe illumination changes for images taken under various conditions. Comparison with other approaches based on color constancy algorithms show that the proposed method achieves the best segmentation results while requiring the least computation time. The simplicity and the robustness of the method make it suitable for real-time applications such as on-board drivers assistance systems. Our future work include using the proposed method as a first step of a road signs recognition system.

\section{REFERENCES}

Barnard, K., Martin, L., Coath, A., and Funt, B. (2002). A Comparison of Computational Color Constancy Algorithms - Part II: Experiments with Image Data. IEEE Transactions on Image Processing, 11(9):985-996.

Bascon, S. M., Arroyo, S. L., Jimenez, P. G., Moreno, H. G., and Ferreras, F. L. (2007). Road Sign Detection and Recognition Based on Support Vector Machines. IEEE Transactions on Intelligent Transportation System, 8(2):264-278.

Benallal, M. and Meunier, J. (2003). Real-time Color Segmentation of Road Signs. In Proceedings of Canadian Conference on Electrical and Computer Engineering (IEEE CCECE 03), pages 1823-1826.

Ebner, M. (2004). Color Constancy Using Local Color Shifts. In Proceedings of the European Conference on Computer Vision, pages 276-287.

Escalera, A., Armingol, J. M., and Mata, M. (2003). Traffic Sign Recognition and Analysis for Intelligent Vehicles. Image and Vision Computing, 21:247-258.

Fang, C. Y., Chen, S. W., and Fuh, C. S. (2003). Road Sign Detection and Tracking. IEEE Transactions on Vehicular Technology, 52(5):1329-1341.

Finlayson, G., Drew, M., and Lu, C. (2004). Intrinsic Images by Entropy Minimization. In Proceedings of the European Conference on Computer Vision, pages 582-595.

Finlayson, G. D., Schiele, B., and Crowley, J. L. (1998). Comprehensive Colour Image Normalization . In Proceedings of the European Conference on Computer Vision, pages 475-490.

Fleyeh, H. (2004). Color Detection and Segmentation for Road and Traffic Signs. In Proceedings of the 2004 IEEE Conference on Cybernetics and Intelligent Systems, pages 808-813.

Fleyeh, H. (2005). Traffic Signs Color Detection and Segmentation in Poor Light Conditions. In MV A2005 IAPR Conference on Machine Vision Applications, pages 306-309.

Gao, X., Podladchikova, L., Shaposhnikov, D., Hong, K., and Shevtsova, N. (2006). Recognition of traffic signs based on their colour and shape features extracted using human vision models. Journal of Visual Communication and Image Representation, 17(4):675-685.

Le, T. T., Tran, S. T., Mita, S., and Nguyen, T. D. (2010). Real Time Traffic Sign Detection Using Color and Shape-Based Features. In Proceedings of Intelligent Information and Database Systems, Second International Conference, pages 268-278.

Paclik, P., Novovicova, J., Pudil, P., and Somol, P. (2000). Road Sign Classiffication Using Laplace Kernel Classifier. Pattern Recognition Letters, 21:1165-1173.

Zakir, U., Zafar, I., and Edirisinghe, E. A. (2011). Road Sign Detection and Recognition by Using Local Energy based Shape Histogram (LESH). International Journal of Image Processing, 4(6):567-583. 